\title{
La voluntad del retorno: correspondencia desde el exilio catalán*
}

\author{
GEMMA CABALLER \\ Y \\ QUERALT SOLÉ \\ Universitat de Barcelona, Centre d'Estudis Històrics \\ Internacionals
}

\begin{abstract}
Estimat amic: Tot allò trist que prevèiem ha vingut-i potser més dolorosament, almenys més precipitadament que no prevèiem. Som nombrosos els escriptors i els universitaris que pocs dies o poques hores abans de la caiguda de Barcelona en varem sortir, per a passar la frontera quan i com poguérem, a través de tota mena de perills i penalitats. Jo sóc ara de moment, amb la meva dona i els meus tres fills, a Avinyó, gaudint de la commovedora hospitalitat els amics provençals. He estat, en totes les peripècies de la nostra odissea, dels més sortosos. ${ }^{1}$
\end{abstract}

Numerosos libros de historia se han basado y han profundizado sobre la temática del exilio. La bibliografía respecto este fenómeno es extensa, pero a pesar de los rigurosos estudios académicos, estos en su mayoría han dejado forzosamente atrás en sus análisis globales los sentimientos personales que hizo aflorar el exilio: los miedos,

Este artículo se enmarca en el proyecto de investigación 'El franquismo en Cataluña. Institucionalización del régimen y organización de la oposición (19381979) [2]', (HAR 2009-1079), financiado por el Ministerio de Ciencia e Innovación.

Esta es la versión aceptada de las autoras del artículo publicado en la revista Bulletin of Spanish Studies, Vol.89, Iss. 7-8, p. 201-213, 2012, disponible en

http://dx.doi.org/10.1080/14753820.2012.731568

1 'Estimado amigo: Todo aquello triste que preveíamos ha venido-y quizás más dolorosamente, al menos más precipitadamente que no preveíamos. Somos numerosos los escritores y los universitarios que pocos días o pocas horas antes de la caída de Barcelona salimos, para pasar la frontera cuando y como pudimos, a través de todo tipo de peligros y penalidades. Yo estoy ahora de momento, con mi mujer y mis tres hijos, en Aviñón, disfrutando de la conmovedora hospitalidad [de] los amigos provenzales. He estado, en todas las peripecias de nuestra odisea, de los más afortunados' (carta del escritor Carles Riba desde Aviñón, 13 de febrero de 1939, al editor Joan Gili, en Londres -texto traducido del catalán por las autoras. Fondo Personal [FP] Carles Riba. Arxiu Nacional de Catalunya [ANC]). 
deseos, esperanzas, preocupaciones, ánimos, etc. de todas aquellas personas que se vieron obligadas a abandonar un país ocupado después de una larga guerra civil. Cientos de miles de hombres, mujeres y niños, cientos de miles de derrotas, cientos de miles de emociones, un único exilio: la huida obligada de Cataluña y la esperanza de regresar pronto a su país.

En este texto, apenas el principio de una larga investigación, queremos ir más allá de la visión que se nos ha ofrecido del fenómeno: estos centenares de miles de personas pueden ofrecernos una visión más personal, más completa y más fiel del que fue un fenómeno tan complejo. Para conseguirlo nos hemos basado en una fuente que consideramos que hasta el momento no se ha utilizado suficientemente: los epistolarios. A través de ellos podemos ofrecer otra visión del exilio que hasta ahora no se había tocado con profundidad: sensaciones, miedos, incertidumbres, esperanzas, inquietudes, etc., que rodearon a personas de diversas tendencias que se vieron obligadas a huir hacia el exilio pero que compartían una voluntad común: el retorno.

El estudio de los epistolarios es una tarea compleja y pesada, en especial cuando se recurre principalmente, como ha sido nuestro caso, a la consulta de fuentes primarias depositadas en fondos de archivos diversos-Arxiu Nacional de Catalunya, Arxiu Històric de Terrassa, Biblioteca del Pavelló de la República (Universitat de Barcelona), etc.-, aunque también nos han sido útiles los epistolarios editados de una persona o entre dos personajes. Los epistolarios llevan implícito un trabajo importante de transcripción pero, sobre todo, de contextualización, que los hace especialmente complejos, a la par que interesantes. El periodo en el que nos hemos centrado inicialmente es el comprendido entre 1939, cuando se inicia el exilio de aquellas personas que huyen de las autoridades franquistas, y el año 1946, cuando finalizada la II Guerra Mundial la esperanza que la victoria aliada había hecho nacer entre los exiliados se desvanece definitivamente al ver que la derrota del eje en Europa y en el mundo no significa un cambio en la situación política de España.

\section{La situación en Catalunya}

El análisis de los epistolarios nos ha dado la oportunidad de acercarnos a personajes clave de la historia de Cataluña que a través de sus cartas, enviadas o recibidas, nos permiten observar desde la distancia del tiempo las sensaciones, miedos, angustias o esperanzas de quienes, a partir de 1939, tuvieron que huir de su país por miedo a las represalias franquistas, sentimientos que expresaban a amigos y familiares a través de su correspondencia. Como se podrá observar a través de las cartas, a pesar de una imagen bastante homogénea que se 
nos ofrece del exilio a través de sus estudios, existieron mil circunstancias y mil casos diferentes. Algunas de estas personas, como Maurici Serrahimauno de los miembros fundadores de Unió Democràtica de Catalunya (UDC), un partido conservador catalán, con una ideología de derechas y católico, que se mantuvo totalmente fiel a la legalidad republicana dando apoyo al gobierno de la República-, se vieron obligadas a salir de Cataluña cuando el ejército nacional estaba a las puertas de Barcelona, dejando atrás mujer e hijos. El hecho de salir solo, abandonando a la familia, le generó una situación particular, pues padeció una doble lejanía: la de la tierra y la familiar. Otras personas, como el escritor Carles Riba, se marchó con toda su familia y la lejanía de la tierra estuvo paliada por la proximidad de los seres queridos. Riba estuvo pidiendo información sobre la situación en Cataluña durante los primeros años del régimen franquista, dudando si irse a México, Inglaterra, etc., hasta que en 1943 decidió volver a España. En ambos casos-y en muchos más-se destaca que en un primer momento un tema recurrente en la correspondencia de los exiliados era el deseo de conocer como se estaba desarrollando la ocupación del país abandonado, las represalias y la situación de familiares y amigos. La necesidad vital de información era muy grande, como pone de manifiesto esta carta que Maurici Serrahima envía a su amigo Josep Maria Trias Peitx, también exiliado y miembro de UDC, donde explica la situación que se vive en Cataluña: 'Veo por los periódicos que las autoridades barcelonesas son todas catalanas y conocidas-parientes tuyos y todo!- . Y que por otro lado no se ve ningún tipo de renovación. iQué país, Castilla! Siempre serán los mismos. De todos modos, la sensación que sienten el peso del hecho catalán, la tienes,-y que conste que de todo esto no quita ningún tipo de consecuencia-, así como la de que no querrán que oficialmente se hable de país conquistado. De todas formas, yo te diré que tengo unas ganas locas de tener noticias de casa, de la mujer, que se debe encontrar desamparada, de los pequeños, de todos ....'2

Gran parte de la correspondencia pone de manifiesto la incertidumbre y el miedo sobre la situación que se da en Cataluña. La inquietud es total, como demuestran estas palabras del escritor Carles Riba: 'Tengo noticias de allá abajo. Las cosas no funcionan. La represión contra el catalanismo y los catalanistas propiamente dichos-o sea, en el tam [sic] no político-no parece que haya empezado, pero no hay ninguna garantía de que no empiece en cualquier momento, al contrario'. ${ }^{3}$ Maurici Serrahima sufrió también la represión franquista debido a su declarado catalanismo. No podemos olvidar que uno de los miembros de Unió Democràtica de Catalunya fue Manuel Carrasco y Formiguera, ejecutado por los franquistas en Burgos en 1937. Así expresaba su miedo y el que sentía por otros

\footnotetext{
$2 \quad$ Carta de Maurici Serrahima desde Burdeos, 3 de febrero de 1939, a Josep M. Trias Peitx, en París. Fondo Personal Trias Peitx, Biblioteca del Pavelló de la República (Universitat de Barcelona), a partir de ahora FP Trias Peitx (BPR-UB) (texto traducido del catalán por las autoras).

Carta de Carles Riba desde el Moulin du Château de Bierville, Boissy-laRivière, 19 de marzo de 1939, a Francesc Trabal, también exiliado en Francia. FP Carles Riba (ANC) (texto traducido del catalán por las autoras).
} 
miembros de UDC en otra carta enviada a Josep Maria Trias Peitx: 'Ya sabrás por Lluís las nuevas de Barcelona, y la detención de Duran, después de haber sido "depurado" y repuesto en su cargo, gracias a una gamberrada de Junyent. Es interesante el hecho de que nadie me haya buscado, y que Coll y otros estén también tranquilos; me ha transmitido alegría, porque aleja la posibilidad de problemas para los de casa. Ahora, por mi caso, eso sólo tiene un valor informativo, lo que quiere decir que si me hubiera quedado en Barcelona quizás nadie hubiera dicho nada, quizá! porque yo tal vez era más conocido que los demás. Tanto da. Pero como estoy aquí, y por estar aquí tengo que volver, y no puedo pasar desapercibido ni que quisieran hacerse los distraídos, el caso ya no es lo mismo. Creo que es la solución que se dé al caso Duran en la que podemos fijarnos; sea lo que quiera el motivo de la denuncia, la actuación política deberá salir, y entonces tendremos una declaración oficial sobre la situación de nuestra gente. Dios haga que sea buena por Duran y por todos los demás que se han quedado. Después, con calma, veremos lo que pueda significar para los que estamos aquí, ${ }^{4}$ o bien '[s]e ve que por ahora no ha habido nada contra los de Unió de una manera directa: de casa me lo confirman en la última carta, que recibí cuando acababa de escribir tu anterior. Eso me tranquiliza por los de casa y también contribuye el hecho de que están en relación más íntima que nunca con el primo ministro: Rafael ha pasado por Bilbao en viaje de bodas y ha estado en casa de él, etc. Si por ellos hubiera dificultades, tendríamos buenos padrinos [...]. También llaman de casa [informando] que mi primo Alejandro Pozos es cónsul de España en Cannes. Le he escrito dos palabras'. ${ }^{5}$

Se puede afirmar que la preocupación por la situación en Cataluña está presente en todos los exiliados, no sólo en los miembros de los partidos políticos, como muestran estas líneas del pintor Joan Miró: 'Hemos recibido una postal de la esposa de Joan Prats. Su hijo ha desaparecido en el frente del Ebro. De la forma en que está escrita esta postal, nos tememos que algo serio haya pasado a nuestro amigo Joan. Tengo noticias que la represión allí es muy dura. Estoy contento de qué por fin su cuñado haya podido salir del campo, esté contento ahora con vosotros' ${ }^{6}$ o esta posterior, también del pintor: 'Esperamos, sin embargo, que a no muy tardar le podremos ver de nuevo en nuestra casa, las noticias que yo tengo de allá abajo son muy malas, y vos ¿qué sabéis? Prats ya está liberado, pero sufrió muchísimo'. ${ }^{7}$

\footnotetext{
Carta de Maurici Serrahima desde Burdeos, 19 de mayo de 1939, a Josep M. Trias Peitx, en París. FP Trias Peitx (BPR-UB) (texto traducido del catalán por las autoras).

$5 \quad$ Carta de Maurici Serrahima desde Burdeos, 7 de agosto de 1939, a Josep M. Trias Peitx, en París. FP Trias Peitx (BPR-UB) (texto traducido del catalán por las autoras).

$6 \quad$ Carta de Joan Miró desde París, 20 de abril de 1939, al arquitecto Domènec Escorsa, también en Francia. FP Domènec Escorsa (ANC) (texto traducido del catalán por las autoras).

Carta de Joan Miró desde París, 28 de enero de 1940, Varengeville sur Mer, al arquitecto Domènec Escorsa, también en Francia. FP Domènec Escorsa (ANC) (texto traducido del catalán por las autoras).
} 
Esta preocupación también se puede observar en una carta que el político Antoni Rovira i Virgili envía al periodista Francesc Trabal el 29 de mayo de 1939 donde le explica que '[h]an llegado algunos fugitivos recientes de Cataluña, que pintan la situación económica y política con los más negros colores'; ${ }^{8}$ o esta carta posterior que Antoni Rovira i Virgili envía al también político Josep Conangla y Fontanilles el 12 de agosto de 1939, donde le explica las noticias que ha recibido de Barcelona: 'La parte de la familia de mi mujer que quedó en Cataluña ha sido muy desdichada. Mi cuñado Sebastián ha sido condenado a 30 años de reclusión por ser republicano y catalanista, una sobrina, a 15 años, iy tiene un hijito de dos años!; mi cuñado Ramón se suicidó en Tarragona el mes pasado por todas estas cosas, y los hijos pequeños quedan en triste situación'. ${ }^{9}$ Una buena muestra también es esta carta del escritor Carles Riba a su amigo Joan Gili: 'Nuevas de allí abajo? Muy tristes. Hambre y todo tipo de arbitrariedades. No hace mucho he recibido por primera vez de allí abajo la advertencia categórica de no volver. Saben de un modo positivo que lo hubiera pasado "muy mal" si me hubieran encontrado. Como escritor, soy el enemigo público número 1-cosa que me honra extraordinariamente, yo que siempre, durante la guerra, he hablado y escrito en el tono de conciliación que tiene aún el artículo editorial de la RC al que me refería más arriba, y por eso le recordaba la paternidad. Mis obras han sido prohibidas-ilo que me revancha deliciosamente de mi fama de incomprensible! No sabéis, pues, qué delito cometéis, Elisabeth y Joan, cuando me leéis'. ${ }^{10}$

Otro problema recurrente reflejado en la correspondencia, sobre todo en los primeros momentos del exilio, es como poder mantener el contacto con la gente de Cataluña, ya que esto no siempre es posible: '¿Hay manera de escribir a Barcelona? [...] He ido a la Rue St. Remi y allí me han dicho que era necesario que viese, por las cosas que hacen referencia a Cataluña, un tal M. Dupoy que vive en la Rue Palais-Gallieu n. 26. He ido varias veces sin encontrarlo: le he dejado una nota y mañana por la mañana intentaré llamarlo: ya te diré una cosa u otra'; ${ }^{11}$ o “El “correo especial” de allá abajo se nos ha agotado, al menos de momento, y puedes contar con que eso no contribuye a ponernos de más buen humor, pero como por otro lado hemos tenido por correo ordinario las noticias esenciales y sabemos que todo el mundo está bien, tampoco nos hemos preocupado más de la cuenta'. ${ }^{12}$ Las cartas también hacen referencia a sistemas 'alternativos' que se inventan los exiliados para recibir información de su casa: 'En este momento recibo carta y fotografías de casa, por un medio que sólo sirve para una vez, pero

$8 \quad$ Antoni Rovira i Virgili, Cartes de l'exili: 1939-1949 (Barcelona: Publicacions de I'Abadia de Montserrat, 2002), 54 (texto traducido del catalán por las autoras).

9 Rovira i Virgili, Cartes de l'exili, 46 (texto traducido del catalán por las autoras).

$10 \quad$ Carta de Carles Riba desde Aviñón, 30 de noviembre de 1939, a Joan Gili, en Londres. FP Carles Riba (ANC) (texto traducido del catalán por las autoras).

$11 \quad$ Carta de Maurici Serrahima desde Burdeos, 3 de febrero de 1939, a Josep M. Trias Peitx, en París. FP Trias Peitx (BPR-UB) (texto traducido del catalán por las autoras).

$12 \quad$ Carta de Maurici Serrahima desde Burdeos, 1939, a Josep M. Trias Peitx, en París. FP Trias Peitx (BPR-UB) (texto traducido del catalán por las autoras). 
nunca más!'13 O bien '[h]e recibido, estos días, "por la puerta falsa", un montón de letras de Barcelona: estudiantes, licenciados jóvenes, etc. Es conmovedor como sufren: algunos excluidos de bibliotecas y universidades, y todos sintiéndose en un ambiente de invasión (no se trata de extremistas!), de hastío, de horror (palabras literales!) pero también como se defienden con armas espirituales y como esperan de ellos mismos y de nosotros. Una palabra nuestra de aliento que les llegue, en prosa o en verso, circula por pequeños "grupos" y tiene una resonancia inmensa. En toda la carrera de escritor y de profesor no había tenido una satisfacción similar. He encontrado, al parecer, un "medio" para hacerles llegar la revista. Esto quiere decir, contando con los defectos y fallos de toda obra humana, el bien que hacemos con nuestras empresas culturales de emigrados. Qué bien puede hacer usted con la revista que proyectan. [...] ${ }^{14}$ Del mismo modo, las cartas también reflejan la importancia de hacer llegar, además de información, objetos materiales o dinero a Catalunya: 'Tú sabes si se pueden cursar dinero y paquetes en Barcelona? No sé donde enterarme'. ${ }^{15}$

Otro tema clave de las informaciones que llegan de Barcelona son las confiscaciones. Pau Casals, por ejemplo, en una carta que envió a Boaz Piller, entonces director de la Orquesta Sinfónica de Boston (6 de mayo de 1940), le explicaba que en España todos sus bienes habían sido confiscados. ${ }^{16}$ No fue el único. En una carta que Antoni Rovira i Virgili envía a Josep Conangla y Fontanilles el 12 de agosto de 1939 éste le explica las noticias que ha recibido de Barcelona: 'Por nuestra parte hemos perdido todo lo que teníamos como resultado, más bien magro, de una vida de trabajo. El chalet de Barcelona, que había yo pagado a plazos durante veinte años, me ha sido confiscado, y allí tuve que dejar mis libros y papeles. Pero yo no estoy desalentado. Sinceramente te digo que espero, a no tardar mucho, una noble revancha para Cataluña y los catalanes'. ${ }^{17}$

\section{Miedo a represalias}

Otro tema clave para algunos de los exiliados, y que se refleja claramente en la correspondencia de determinados personajes, es el miedo a hacer cualquier actividad que afecte a la familia que se ha visto obligada a quedarse en Catalunya. Este es un tema recurrente de una parte de los refugiados catalanes, especialmente de aquellos que por sus vinculaciones políticas durante la guerra tenían, una vez en el exilio, la posibilidad de

13 Carta de Maurici Serrahima desde Burdeos, 4 de agosto de 1939, a Josep M. Trias Peitx, en París. FP Trias Peitx (BPR-UB) (texto traducido del catalán por las autoras).

14 Carta de Carles Riba desde Isle-Adam, 22 de abril de 1940, a Joan Gili, en Londres. FP Carles Riba (ANC) (texto traducido del catalán por las autoras).

$15 \quad$ Carta de Maurici Serrahima desde Burdeos, 17 de marzo de 1939, a Josep M. Trias Peitx, en París. FP Trias Peitx (BPR-UB) (texto traducido del catalán por las autoras).

16 Pau Casals y Josep Trueta, Estimat doctor/Admirat mestre: l'esperit d'una amistat en 79 cartes, ed. Quim Torra (Barcelona: Acontravent, 2009), 36.

17 Rovira i Virgili, Cartes de l'exili, 46 (texto traducido del catalán por las autoras). 
llevar a cabo alguna actividad de tipo político. Existe un miedo evidente a las consecuencias que en el interior podría tener cualquier acción realizada en el primerísimo exilio, tanto por la influencia negativa que podría comportar para su posible regreso como las consecuencias que podría acarrear a la familia que se había quedado en España. Para Maurici Serrahima este fue un tema clave: 'Por ahora, los que hemos dejado allí abajo mujer e hijos creo que tenemos derecho a un silencio para no perder la posibilidad de sacarlos así que podamos mantenerlos'; ${ }^{18}$ o 'Además, no hay que olvidar que tenemos las familias allí dentro, y que debemos tener la discreción necesaria para que no se nos estropeen las probabilidades de sacarlas. Lo digo muy en serio, y pienso que con ello no se perjudica nada nuestra política, y en cambio se mejorará mucho nuestra-la mía, menos libertad para hacerla. Otra cosa, por medio de Clara, o de quien sea, ise puede escribir a Barcelona? Se puede recibir alguna novedad? [...] Hace ocho días, y no sé nada: no me impaciento, pero tengo muchas, muchas ganas de tener noticias. Ya me dirás si tienes nada pensado'. ${ }^{19}$ Esta postura estaba claramente relacionada al hecho que las informaciones que llegaban de Barcelona en los primeros momentos eran desoladoras. Una buena muestra es la información que la mujer de Maurici Serrahima envía a su marido: '[Mi mujer] [m]e pide con una insistencia desesperada que no haga política. Se muestra desolada y hastiada de la actitud sanguinaria y vengativa de los franquistas que llegan. Me hace ver muy claro que, si les pasara algo, no podría contar para nada con esta gente: hasta los más amigos tienen miedo de comprometerse. Me habla de venir. E insiste con verdadero fervor que, sobre todo, no haga política. Comprendo lo que me quiere decir, claro, que cualquier acto público mío puede costarle muy caro a ella y a toda la familia. (Ayer hablé con un vasco que, sólo porque aquí fue del Comité de los refugiados, tuvo la mujer ochenta días prisionera en Bilbao.) Ya entiendes lo que quiero decir, con todo esto. Tenemos ganado el derecho al silencio, y estoy decidido a utilizar este derecho. Sobre todo porque sería estúpido jugarse cosas que son sagradas por el gusto de hacer politiquilla, de hacer el trasiego por Paris. No me da la gana, por pequeñas tonterías, de perjudicar en absoluto la gente de allí abajo. Que pena tienen de no respirar una atmósfera libre como la que respiramos nosotros. Somos todos los que tenemos que callar y que debemos estar quietos una temporada: los unos por necesidad absoluta, como yo, otros por respeto a nuestro caso. Y creo que, políticamente, este silencio es la mejor cosa que podemos hacer. Sabes muy bien que esto no es un retroceso: si fuera necesario para las cosas esenciales, yo siempre he estado y estaré en mi sitio. Pero ahora no hay nada que pueda molestar al silencio, más importante que cualquier actividad política [...]. No hace falta que te diga que, por mi parte, no quiero aparecer por ningún lado ni quiero que mi nombre suene para nada'. ${ }^{20}$ Este es un tema que atormentaba profundamente Maurici Serrahima: 'Yo no puedo estar allí [en un encuentro

$18 \quad$ Carta de Maurici Serrahima (¿desde Burdeos, febrero de 1939?), al político Joan B. Roca Caball. FP Trias Peitx (BPR-UB) (texto traducido del catalán por las autoras).

$19 \quad$ Carta de Maurici Serrahima desde Burdeos, 31 enero de 1939, a Josep M. Trias Peitx, en París. FP Trias Peitx (BPR-UB) (texto traducido del catalán por las autoras). 
del Consejo Nacional Católico] porque no puedo enseñarme, por [xxx] de los que están allí abajo-no por un eventual retorno mío, en el que ya sabes que no acabo de creer ...'.21

\section{Carestía y añoranza}

A la situación de tristeza e incertidumbre se unían también las duras condiciones y las carencias logísticas. Así, las cartas que los exiliados envían a sus familiares contienen noticias del hambre que padecen, de la falta de combustible y de comida. En muchos casos las cartas nos permiten conocer las condiciones de personas exiliadas que no se encontraban en los campos de refugiados, aunque no por ello esta circunstancia era un consuelo para algunos. Las pocas cartas que hasta el momento se han localizado de internos en los campos de refugiados franceses muestran la voluntad de animar a la familia que reside en Cataluña. En este sentido consideramos especialmente interesante la correspondencia de Jaume Pons a su familia de Bellpuig d'Urgell, depositada en el Arxiu Nacional de Catalunya. Jaume Pons Escolà (1914-1941) ocupó durante los años 1933 a 1939 el cargo de secretario del Ayuntamiento de Les Borges Blanques. En 1939 marchó a Francia y fue recluido en un campo de concentración. Destaca especialmente de las cartas el hecho de que resulte sorprendente que casi nunca explique nada suyo y en cambio pida constantemente información de familiares, vecinos, amigos, a qué se dedican, qué hacen, cómo va la vida por el pueblo, etc. Por parte de Jaume Pons se percibe una gran añoranza de su pueblo, su tierra y su familia. Pregunta y habla del tiempo, si toca ir a segar, y les pide a menudo que estén tranquilos, que vendrán tiempos mejores y que él está muy bien, suponemos que para no preocuparles. La voluntad de no angustiar a la familia se refleja claramente en esta carta enviada desde el campo de Barcarés en abril de 1939: 'Por aquí llevamos una vida muy aburrida, solo comiendo y descansando, pero como estamos muchos del pueblo, aún nos lo vamos pasando bien; además recibimos noticias de los del Canat, Farré, Nou, Francisco y Juan Nabau, A. Monturiol, José Areny y su hermana Magdalena, Celestino Rosell [...]. Observando y remarcando las diferencias que existen entre esta vida y la que pasábamos en el pueblo casi todo el día reunidos y así nos parece más llevadera nuestra vida por las tierras extranjeras'; ${ }^{22}$ o también '[m]i vida por aquí sigue como siempre bastante satisfactoria pues el trabajo no es pesado y la alimentación buena. Además por aquí tenemos muchas diversiones y esto hace que pasemos la vida bastante divertida. La semana pasada con Ginestà, fuimos a un festival que dio una compañía de artistas españoles que resultó muy variado y divertido, en el cual estábamos al paso de veinte

\footnotetext{
$20 \quad$ Carta de Maurici Serrahima desde Burdeos, 14 de febrero de 1939, a Josep M. Trias Peitx, en París. FP Trias Peitx (BPR-UB) (texto traducido del catalán por las autoras).

$21 \quad$ Carta de Maurici Serrahima desde Burdeos, 10 de junio 1939, a Josep M. Trias Peitx, en París. FP Trias Peitx (BPR-UB) (texto traducido del catalán por las autoras).

$22 \quad$ Carta de Jaume Pons Escolà desde Barcarés, 29 de abril de 1939, a su familia, en Bellpuig d'Urgell (Lleida). FP Jaume Pons Escolà (ANC) (texto traducido del catalán por las autoras).
} 
mil espectadores. Ahora he entrado a trabajar junto con Molins en una farmacia-hospital donde él trabaja de "charpentier" y yo de "charpentierelectricista". Estoy muy impresionado pues me parece que estaré muy bien' ${ }^{23}$ Pese a los intentos, la añoranza se desprende en esta carta enviada dos meses después: ‘[...] También veo que habéis celebrado regularmente la célebre fiesta mayor de nuestro pueblo y esto me alegra bastante aunque reconozco que no podía ser tan lucida como los años anteriores, ya que las anómalas circunstancias que hoy concurren en nuestra España impiden que permanezcan en su pueblo los jóvenes que son los que alegran tanto las fiestas. [...] Yo, por mi parte sigo muy bien y en el mismo lugar de siempre, con los compañeros del pueblo que ahora formamos un grupo de 12 y estamos todos muy bien unidos [...] A Manuel le digo que si aquí casi no hay chicas [se refiere a Cataluña], es peor donde estoy yo, que todo son hombres, y no hay ninguna, y fuera de algún domingo que tenemos visita, nunca vemos ninguna'. ${ }^{24}$

Estrechamente relacionado con este tema están todas las gestiones que reflejan las cartas para intentar facilitar información de cómo sacar familiares y amigos de los campos de concentración. Una buena muestra de ello es la carta que Joan Miró envió a Domènec Escorsa: 'Amigo Escorsa; perdonad si no he respondido antes a su letra, pero esperaba poder darle noticias más concretas. Me he enterado sobre qué hay que hacer para sacar a su cuñado del campo de concentración, tiene que insistir, pues me dicen que teniendo ya una autorización de un prefecto no le será difícil, ya que hay solamente que seguir los trámites oficiales, siempre largos y engorrosos. Me aconsejan también que se dirija a la compañía consignataria que ha retenido a su cuñado el billete [...]. He pasado también todas estas indicaciones a Picasso, por si puede hacer algo'. ${ }^{25}$

\section{Un futuro incierto}

Finalmente, un tema especialmente presente en la correspondencia de los exiliados es el decidir qué hacer en el futuro inmediato. Así, un tema clave en las cartas son las dudas sobre si quedarse en Francia-lugar que acogió en un primer momento los exiliados-, volver a Cataluña o continuar el camino del exilio más allá de territorio francés. Esta última opción, elegida por muchos de ellos, fue la que siguieron también muchos de los exiliados que en un primer momento optaron por quedarse en Francia, debido al estallido de la Segunda Guerra Mundial y el avance de las tropas alemanas por el territorio francés. En estas circunstancias, la incertidumbre se fue apoderando de los estados de ánimo y muchos optaron por abandonar Francia y proseguir su camino. Esta situación se refleja claramente en la

23 Carta de Jaume Pons Escolà desde Barcarés, 3 de julio de 1939, a su familia, en Bellpuig d'Urgell (Lleida). FP Jaume Pons Escolà (ANC) (texto traducido del catalán por las autoras).

$24 \quad$ Carta de Jaume Pons Escolà desde Barcarés, 24 de junio de 1939, a su familia, en Bellpuig d'Urgell (Lleida). FP Jaume Pons Escolà (ANC) (texto traducido del catalán por las autoras).

${ }_{25}$ Carta de Joan Miró desde París, 22 de marzo de 1939, a Domènec Escorsa, también en Francia. FP Domènec Escorsa (ANC) (texto traducido del catalán por las autoras). 
correspondencia que Joan Miró envía a Domènec Escorsa el 27 de julio de 1939: 'Comprendo sus angustias al ver que se alarga su estancia aquí. Es de suponer podrá salir con la próxima expedición. Yo de todo corazón le ayudaría, pero me encuentro ahora en momentos un poco difíciles, pues últimamente he hecho todo lo que me ha sido posible por otros compañeros nuestros que se encuentran en una situación bastante difícil. Además de esto me encuentro que al regresar a París necesito instalarme y cambiar de piso. No desaniméis, insistid con Pont, la Generalitat bien tiene el deber de hacer que puedan embarcar lo más pronto posible'. ${ }^{26}$ La misma situación de incertidumbre se refleja en la correspondencia de Carles Riba. Éste mantiene contactos con diversos amigos para saber cuál era la situación en Cataluña, como demuestra esta carta a Ramon Sugranyes de Franch: 'Quiero decir que veo tan insegura la situación, tan probable que de un día a otro no tengamos opción, que es de elemental prudencia mantener los amigos de allá abajo en estado de alerta preparados a recibirnos y ayudarnos. [...] Hace pocos días me ha llegado un nuevo mensaje confidencial, a través de JM Casanova, que en resumen dice: Hay muchos, que me son amigos a toda prueba; unos pocos, interesados en molestarme. No es lo que he hecho que me pondría en peligro, tanto como lo que soy, mi nombre etc. El hecho de ser una bandera. Que me espera. No tengo todavía ninguna prisa. ¿Podré hablar así mucho tiempo?'27 Posteriormente escribirá a su amigo Joan Gili: 'Continuamos en Isle-Adam, pero a través de indecisiones y de luchas internas, hemos concluido de no movernos de ella, si no es a París ... io a Londres! Ya sabéis como nuestra venida a Inglaterra era inminente: la residencia, parece estaba poco menos que a punto. La guerra lo ha aplazado sine die. Ha habido momentos que hemos debatido si irnos a América. De México nos tientan, nos hacen ofertas. Uno de nuestros chicos incluso se indignaba porque vacilábamos ... Todo sopesado, es mejor resistir aquí: se encuentra más dentro de nuestro gusto y de nuestro deber, aquí o en Inglaterra podemos sentar raíces, hacer una labor de resultados inmediatos, y la hacemos: la Fundación Ramon Llull está en marcha'. ${ }^{28}$ Al cabo de unos meses hará la siguiente reflexión a Sugranyes de Franch: "En el problema concreto del retorno o de la "permanencia": todo lo que queda entre lo que conseguimos hacer según nuestro esfuerzo recortado por nuestra debilidad, y lo que podríamos llamar la actitud ideal según los principios absolutos, puede ser compensado, y debe serlo, por un nuevo esfuerzo, dentro de las nuevas circunstancias'. ${ }^{29}$

Carles Riba, finalmente, decidirá volver a Cataluña, como le explica al escritor Armand Obiols: ‘Es absurdo, con la alegría que me hizo su letra, que

$26 \quad$ Carta de Joan Miró desde Varengeville sur Mer, 27 de julio de 1939, a Domènec Escorsa, también en Francia. FP Domènec Escorsa (ANC) (texto traducido del catalán por las autoras).

$27 \quad$ Carta de Carles Riba desde Montpeller, 14 de mayo de 1939, a Ramon Sugranyes de Franch, entonces en París. FP Carles Riba (ANC) (texto traducido del catalán por las autoras).

${ }_{28} \quad$ Carta de Carles Riba desde Isle Adam, 30 de noviembre de 1939, a Joan Gili, en Londres. FP Carles Riba (ANC) (texto traducido del catalán por las autoras).

$29 \quad$ Carta de Carles Riba desde Montpeller, 2 de junio de 1941, a Ramon Sugranyes de Franch, entonces en París. FP Carles Riba (ANC) (texto traducido del catalán por las autoras). 
haya tardado tanto en responderte. $Y$ hoy lo hago a toda prisa para deciros ... También quizás es absurdo: volvemos a casa. Lo decidimos a finales de octubre; los trámites, el cierre de la frontera, mover nuevamente la documentación, la incomunicación casi total con la familia, etc. todo esto lo ha hecho arrastrar, y nos ha tenido enervados en una interinidad potenciada. Hoy, de repente, la perspectiva de tener que dejar Montpellier en un plazo breve, y más o menos a la fuerza, no nos deja lugar a más vacilaciones ni esperas. A mediados de la semana entrante deberemos hacer el cabezazo. Nos dejaremos llevar por la ola que nos viene a tomar. Dios quiera que sea suave. Le tengo por demasiado inteligente para ponérseme a especificar en cuenta las razones en pro y en contra que he sopesado, y no conmigo solo. Uno se perdería entre principios puros; vale más situarse en la realidad imperfecta y pensar que sólo hay situaciones y posibilidades individuales, y que en todas y según todas, se puede contribuir a salvar los principios. No se me ha pedido nada que signifique retractación, ni en pensamiento, y voy allá abajo en las condiciones del más oscuro de los emigrados que vuelve, expuesto a tener que padecer alguna experiencia poco agradable y nueva para mí. No será inútil. [...]'.30

La voluntad de retorno estuvo presente en el exiliado desde el mismo instante en que pisó tierra extranjera. Pero esta voluntad de retorno no se limitó, lógicamente, a los primeros tiempos. Quizás uno de los momentos de máxima esperanza de retorno por parte de los españoles exiliados fue al final de la Segunda Guerra Mundial, con el triunfo de las democracias. Y uno de los máximos exponentes del desencanto que causa la respuesta no intervencionista de las democracias aliadas hacia la dictadura franquista-por no decir el máximo exponente-es el caso de Pau Casals. El violonchelista Pau Casals mantuvo con el médico Josep Trueta una extensa correspondencia entre 1939 y 1973 recogida en el libro Estimat doctor/Admirat mestre: l'esperit d'una amistat en 79 cartes. En una carta de Casals a Trueta escrita la Navidad de 1944 Casals manifestaba: 'Sí, pronto nos podremos abrazar en nuestra casa. A medida que se acerca este inefable momento aumenta el deseo y la impaciencia, al punto de no poder pensar en nada más. Es así que antes de hacer planes de viajes por Europa, siento la absoluta necesidad de reencontrar Cataluña, ver los míos y mi casa de San Salvador [...]. Envidio los catalanes que se encuentran en esta nunca suficientemente querida y admirable Inglaterra. Yo soy, porque la conozco, de los que no han dudado de ella en ningún momento. Que tomaría, a costa de sacrificio máximo, la responsabilidad y la protección de los otros pueblos, consciente de su grandeza en todos los sentidos'. ${ }^{31}$ Este sentimiento era compartido por Josep Trueta-que residía en Oxford-en una carta que envía a Casals en marzo de 1945: 'Como tú, querido maestro Casals, tengo que poner freno a los impulsos que a menudo emergen de dentro de mí haciéndome sentir el deseo desgarrador de ver la Tierra. Qué lejos estoy'. ${ }^{32}$ Ya terminada la Segunda Guerra Mundial las esperanzas siguen como muestra esta carta de Trueta a Casals de diciembre de 1945:

\footnotetext{
$30 \quad$ Carta de Carles Riba desde Montpeller, 26 de marzo de 1943, a Armand Obiols, también en Francia. FP Carles Riba (ANC) (texto traducido del catalán por las autoras).

31 Casals y Trueta, Estimat doctor/Admirat mestre, 41 (texto traducido del catalán por las autoras).
} 
'Preparados ya para celebrar la última Navidad del exilio entre las nieblas húmedas de Oxford, le recordamos con afecto. Usted, querido maestro, aunque no pueda pasar estos días cerca del hogar en San Salvador, tenga el disfrute de pisar tierra catalana y hasta de ver encima de las montañas blancas el cielo de nuestra Catalunya. Que el año que va a empezar nos devuelva a la Patria y nos conserve el afecto'. ${ }^{33}$

Pero todas las esperanzas se rompieron. Los países aliados optaron por no intervenir en España después del fin de la Segunda Guerra Mundial y la decepción de Pau Casals fue enorme, como expresaba en esta carta a Trueta del 17 de noviembre de 1946: 'Los gobiernos de las naciones más responsables nos tratan como cantidad_despreciable hasta el punto de pasar por encima de la moral, la justicia, de todos los sentimientos humanos'. ${ }^{34}$ La decepción e indignación de Pau Casals fue absoluta, lo que supuso su decisión de no volver a interpretar públicamente mientras las democracias no cambiaran su postura hacia España.

Una emotiva muestra de la voluntad del retorno al país forzadamente abandonado es la carta de Felip Barjau, que había sido diputado a las Cortes Españolas por el Partit Socialista Unificat de Catalunya. Un deseo de retorno reiterado en la correspondencia analizada y que se ha convertido en el hilo conductor de nuestra investigación: 'En alejarme de mi país siento una frialdad glacial en mi corazón, que sólo puedo resistir si pienso que este supremo sacrificio puede servir más tarde para liberar nuestra patria de todos los prejuicios ancestrales que la minan, si tenemos el acierto de llevar a cabo una actuación sincera y de cara al devenir. Queda la ilusión de vernos un día hermanados alrededor de nuestra Cataluña para dar lugar a nuestro último homenaje consagrando a nuestros despojos después de haber consagrado toda una vida. El hecho de haber sufrido por ella tantos y tantos sacrificios nos da más derecho a quererla y elogiar las virtudes y los encisos. Os puedo decir, amigo, que nunca había sentido tan intensamente el amor a mi tierra como ahora, cuando todo está decidido por alejarse mas de ella'. ${ }^{35}$

\footnotetext{
32 Casals y Trueta, Estimat doctor/Admirat mestre, 42 (texto traducido del catalán por las autoras).

33 Casals y Trueta, Estimat doctor/Admirat mestre, 49 (texto traducido del catalán por las autoras).

34 Casals y Trueta, Estimat doctor/Admirat mestre, 51 (texto traducido del catalán por las autoras).

$35 \quad$ Carta de Felip Barjau desde Montagnac, 11 de mayo de 1939, a Manuel Serra i Moret, entonces en Surrey (Reino Unido). Fondo personal Manuel Serra i Moret (BPR-UB) (texto traducido del catalán por las autoras).
} 